\title{
CHANGES IN ENERGY METABOLISM OF THE JUVENILE FASCIOLA HEPATICA DURING ITS DEVELOPMENT IN THE LIVER PARENCHYMA
}

\author{
ALOYSIUS G.M. TIELENS, JOSEPHUS M. VAN DEN HEUVEL and SIMON G. VAN DEN BERGH \\ Laboratory of Veterinary Biochemistry, State University of Utrecht, Biltstraat 172, 3572 BP Utrecht, \\ The Netherlands.
}

(Received 23 March 1982; accepted 10 June 1982)

Juvenile Fasciola hepatica at different stages of development were isolated from the liver parenchyma of experimentally infected rats. Their energy metabolism was studied by incubation with $\mathrm{D}-\left[6-{ }^{14} \mathrm{C}\right]$ glucose and compared with that of juveniles isolated immediately after in vitro emergence from the metacercarial cysts. These studies confirmed that freshly excysted juveniles have an aerobic energy metabolism, but are already fully equipped for life in the absence of oxygen. A functioning Krebs cycle was a true characteristic of all parenchymal stages of $F$. hepatica, but its capacity slowly decreased during development. Concomitantly, acetate became the major end product. This formation of acetate was an aerobic process and it was the most important source of energy for the exponentially growing fluke after twelve days of development. It might, therefore, be an important new target for anthelmintics. The decrease in Krebs cycle activity was not caused by a lack of oxygen nor by a decreased capacity of the respiratory chain. The freshly excysted juvenile had endogenous glycogen stores which were degraded during fasting and replenished in the presence of glucose.

Key words: Fasciola hepatica, Energy metabolism, Juvenile stage, Development, Glucose breakdown, Acetate formation.

\section{INTRODUCTION}

The adult liver fluke, Fasciola hepatica, ferments glucose mainly to propionate and acetate, both under aerobic and anaerobic conditions [1]. On the other hand, in vitro excysted juvenile $F$. hepatica have an aerobic energy metabolism. In the presence of oxygen, juveniles oxidize glucose mainly to carbon dioxide, but they can also survive prolonged periods of anaerobiosis during which they excrete propionate and acetate, like the adult [2].

It was uncertain whether the observed aerobic functioning is a true characteristic of juvenile liver flukes or merely the result of the in vitro excystment procedure. After all, the in vitro excysted juvenile $F$. hepatica [3] has not met a number of physical barriers that parenchymal liver flukes have to cope with. Burrowing through the intestinal wall, migration in the abdominal cavity and penetration of the liver capsule have not occurred. It is known that such barriers can influence the biochemical properties of 
parasites: when a schistosomal cercaria penetrates the skin of a host and transforms into a schistosomulum, a bioenergetic metamorphosis occurs [4]. In the development of newly excysted $F$. hepatica to parenchymal liver fluke the situation is probably different. Burrowing through the gut wall is not an essential step in this development: the implantation of in vitro excysted juveniles or of untreated metacercariae in the abdominal cavity results in normal development of the flukes $[5,6]$. Penetration of the host liver is also not necessary for normal development, as $F$. hepatica can also end up as an egglaying adult in the lung [6].

In this paper, evidence is presented that juvenile liver flukes at the early parenchymal stage indeed have a fully aerobic energy metabolism. The question then arises at which moment the transition to the anaerobic metabolism of the adult occurs. Two hypotheses concerning the trigger of such a transition have been forwarded [7]: the predominantly anaerobic environment of the bile duct could force the liver fluke to abandon the aerobic energy metabolism or the growing size of the liver fluke could limit the diffusion of oxygen and so a further aerobic functioning. If the latter hypothesis is correct, the transition from aerobic to anaerobic energy metabolism may already have occurred in the parenchyma.

Experiments presented in this paper showed that at the later parenchymal stages the energy metabolism of the liver fluke indeed became partly anaerobic. However, early in the parenchymal development changes in the energy metabolism occurred which were not caused by a lack of oxygen but by a diminished Krebs-cycle activity. The aerobic breakdown of glucose to $\mathrm{CO}_{2}$ was gradually diminished and replaced by the aerobic production of acetate.

\section{MATERIALS AND METHODS}

Isolation of flukes. The medium ( $\mathrm{pH} \mathrm{7.3)} \mathrm{used} \mathrm{for} \mathrm{the} \mathrm{isolation} \mathrm{of} \mathrm{all} \mathrm{the} \mathrm{stages} \mathrm{of} F$. hepatica contained (mM): $\mathrm{NaHCO}_{3}$ (39), $\mathrm{NaCl}$ (103), $\mathrm{KCl}(5.4), \mathrm{NaH}_{2} \mathrm{PO}_{4}$ (1), $\mathrm{MgSO}_{4}$ (0.8), $\mathrm{CaCl}_{2}$ (1.8), glucose (11) plus $75 \mathrm{mg} / \mathrm{l}$ streptomycin and $75 \mathrm{I} . \mathrm{U} . / \mathrm{ml}$ penicillin. Newly excysted juveniles were obtained by our previously described method for the in vitro excystment of metacercariae and subsequent isolation of juvenile $F$. hepatica [3]. Parenchymal liver flukes were isolated from male Wistar rats (about $180 \mathrm{~g}$ at time of infection) that had been orally infected with about 100 metacercariae each. The 6-days old flukes were obtained by placing the sliced $(1 \mathrm{~cm})$ livers at $38^{\circ} \mathrm{C}$ on a $500 \mu \mathrm{m}$ sieve in a funnel filled with medium and by collecting after $1.5 \mathrm{~h}$ the flukes that had sunk into the funnel. The sliced $(1 \mathrm{~cm})$ livers of 12-, 24-, and 25 -days infected rats were put in a petri dish in medium at $38^{\circ} \mathrm{C}$ and after $1 \mathrm{~h}$ the flukes were isolated with a pipet. All isolated flukes were washed with fresh medium and their incubations were started immediately.

Incubations. Aerobic and anaerobic incubations of $F$. hepatica were carried out as described before [2] , but D-[6- $\left.{ }^{14} \mathrm{C}\right]$ glucose was used instead of $\mathrm{D}-\left[\mathrm{U}-{ }^{14}\right]$ glucose. 
Assays. The analyses of the excreted end products were performed as published earlier [2].

The protein content of the incubations was determined by the method of Lowry et al. [8] with bovine serum albumin as standard after the flukes had been disrupted by ultrasonic treatment.

Glycogen was isolated and hydrolysed according to Hassid and Abraham [9].

Glucose was determined with the GOD-Perid test combination (Boehringer, Mannheim, F.R.G.).

Materials. The metacercariae were a gift from the Parasitology Department of the Central Veterinary Institute (Lelystad, The Netherlands).

D- $\left[6-{ }^{14} \mathrm{C}\right]$ Glucose was obtained from New England Nuclear (Boston, MA, U.S.A.) and from the Radiochemical Centre (Amersham, England).

Ascorbate oxidase was obtained from Boehringer (Mannheim, F.R.G.), streptomycin and penicillin from Flow Laboratories (Irvine, Scotland).

\section{RESULTS}

Growth of the flukes in vivo. After each incubation the number of flukes was counted and the amount of protein was determined. The flukes grew exponentially during their parenchymal development (Table I). The protein content of the newly excysted liver fluke was found to be $230 \mathrm{ng}$, in contrast to our earlier estimate $[2,3,7]$.

End products of glucose breakdown. Prolonged incubations ( $14 \mathrm{~h})$ were necessary due to the limited amount of material that was available. The vast majority (between 75 and 95\%) of the flukes in all incubations survived, as judged by microscopic observation of their mobility. Those in the aerobic incubations were more active.

In order to increase the accuracy of the results, the parasites were incubated with $D$ $\left[6-{ }^{14} \mathrm{C}\right]$ glucose instead of $\mathrm{D}-\left[\mathrm{U}-{ }^{14} \mathrm{C}\right] \mathrm{glucose}$. The advantage of the former substrate is

\section{TABLE I}

Protein content of the developing $F$. hepatica

\begin{tabular}{ll}
\hline $\begin{array}{l}\text { Age } \\
\text { (days) }\end{array}$ & $\begin{array}{l}\text { Protein content } \\
(\mu \mathrm{g} / \text { fluke) }\end{array}$ \\
\hline 0 & $0.23^{\mathrm{a}}$ \\
6 & $1.1 \mathrm{~b}$ \\
12 & $6.8 \mathrm{~b}$ \\
24 & $56.1 \mathrm{~b}$ \\
\hline
\end{tabular}

a See Table IV.

b Average value of all flukes used at this stage. 
that all the labelled $\mathrm{CO}_{2}$ is produced in the aerobic functioning of the Krebs cycle because the $\mathrm{CO}_{2}$ which is formed in the production of acetate and propionate will not be labelled.

The radioactive end products of glucose breakdown, excreted by the parasites into the medium, were determined and the results of incubations with in vitro excysted and 24- or 25-days old flukes are shown in Table II. In Table III the formation of these labelled end products is expressed as percentages of the amount of glucose catabolized to form these end products. After the sequential elution of glucose, acetate, propionate and lactate from the anion-exchange column with $5 \mathrm{mM} \mathrm{HCl}, 2 \mathrm{M} \mathrm{NaCl}$ was used to elute possible other labelled products, like pyruvate or Krebs cycle intermediates. These products, called 'rest' in Table II were not identified and their amounts were calculated on the assumption that they had half the specific radioactivity of glucose. For the calculation of the produced amounts of acetate, propionate and lactate, a specific activity of half that of glucose was used. The specific activity of carbon dioxide was assumed to be one-sixth of that of glucose. In Fig. 1 the results are shown of the aerobic incubations with the four investigated stages of the developing juvenile $F$. hepatica. The amounts of lactate were negligible and are omitted from the figure.

TABLE II

End products of glucose breakdown excreted by $F$. hepatica

\begin{tabular}{|c|c|c|c|c|c|c|}
\hline \multirow[t]{2}{*}{ Flukes } & \multirow{2}{*}{$\begin{array}{l}\text { Incubation } \\
\text { condition }\end{array}$} & \multicolumn{5}{|c|}{ Excretion of end products ( $\mathrm{nmol} / \mathrm{h}$ per $\mathrm{mg}$ protein) } \\
\hline & & $\overline{\mathrm{CO}_{2}}$ & Acetate & Propionate & Lactate & 'Rest'a \\
\hline In vitro & aerobic & 294 & 10 & n.d. & n.d. & 32 \\
\hline excysted & & 286 & 6 & n.d. & n.d. & 24 \\
\hline In vitro & anaerobic & 16 & 201 & 399 & 80 & 64 \\
\hline excysted & & 16 & 191 & 350 & 73 & 76 \\
\hline \multirow{3}{*}{$\begin{array}{l}24 \text { days } \\
\text { old }\end{array}$} & aerobic & 118 & 455 & 205 & 30 & 49 \\
\hline & & 104 & 461 & 210 & 38 & 61 \\
\hline & & 107 & 454 & 177 & 43 & 62 \\
\hline 25 days & anaerobic & 13 & 180 & 440 & 14 & 22 \\
\hline old & & 13 & 217 & 544 & 12 & 35 \\
\hline
\end{tabular}

The flukes (about $1 \mathrm{mg}$ protein per Erlenmeyer flask) were incubated for $14 \mathrm{~h}$ at $38^{\circ} \mathrm{C}$ with D-[6${ }^{14} \mathrm{C}$ ]glucose $(0.5-0.9 \mathrm{Ci} / \mathrm{mol})$. The radioactive end products in the fluke-free medium were analysed. All values are corrected for blank incubations. n.d. = not detectable. Results of separate incubations are presented. In all incubations the total recovery of labelled end products equalled $94-105 \%$ of the radioactive glucose consumed.

a See text. 
TABLE III

Glucose catabolism by $F$. hepatica

\begin{tabular}{llllll}
\hline Flukes & $\begin{array}{l}\text { Incubation } \\
\text { condition }\end{array}$ & \multicolumn{4}{l}{ Product formation (\% of glucose broken down) } \\
\cline { 3 - 6 } & $\mathrm{CO}_{2}$ & Acetate & Propionate & Lactate \\
\hline $\begin{array}{l}\text { In vitro } \\
\text { excysted }\end{array}$ & aerobic & 91 & 9 & - & - \\
In vitro & & 94 & 6 & - & - \\
excysted & anaerobic & 1 & 29 & 58 & 12 \\
24 days & & 1 & 31 & 57 & 12 \\
old & aerobic & 5 & 62 & 28 & 4 \\
& & 5 & 62 & 28 & 5 \\
25 days & & 5 & 64 & 25 & 6 \\
old & anaerobic & 1 & 28 & 69 & 2 \\
\hline
\end{tabular}

a The amount of glucose broken down in each incubation in the formation of $\mathrm{CO}_{2}$, acetate, propionate and lactate was calculated from the results in Table II and put at $100 \%$.

Depletion and replenishment of the endogenous glycogen stores of in vitro excysted juveniles. Freshly excysted juvenile $F$. hepatica are normally isolated and maintained in the presence of $11 \mathrm{mM}$ glucose [3]. In one series of experiments glucose was replaced by $5.5 \mathrm{mM} \mathrm{NaCl}$. The isolated parasites were divided into 6 portions. Their glycogen content was determined: for 2 portions immediately, for 2 portions after an aerobic incubation of $5 \mathrm{~h}$ at $38^{\circ} \mathrm{C}$ in the absence of glucose and for 2 portions after an aerobic incubation of $5 \mathrm{~h}$ at $38^{\circ} \mathrm{C}$ in the absence of glucose, followed by an aerobic incubation of $11 \mathrm{~h}$ at $38^{\circ} \mathrm{C}$ in the presence of $11 \mathrm{mM}$ glucose. The parasites of all incubations retained their vitality, as judged with an inverted microscope during the counting after the incubations. The results of the glycogen determinations, presented in Table IV, show that the in vitro excysted juveniles had endogenous glycogen stores which could be consumed in the absence and replenished in the presence of added glucose.

\section{DISCUSSION}

In this paper the breakdown of added $\mathrm{D}-\left[6-{ }^{14} \mathrm{C}\right] \mathrm{glucose}$ by juvenile liver flukes was studied by analysing the radioactive end products excreted by the flukes into the medium. Previously it could not be excluded that unlabelled endogenous substrates (e.g. glycogen) of the juveniles were catabolized together with the exogenously added radioactive glucose. The results presented in Table IV show that in vitro excysted juveniles consumed $85 \mathrm{pg}$ of glycogen per ng protein in $5 \mathrm{~h}$ when no exogenous substrate was 


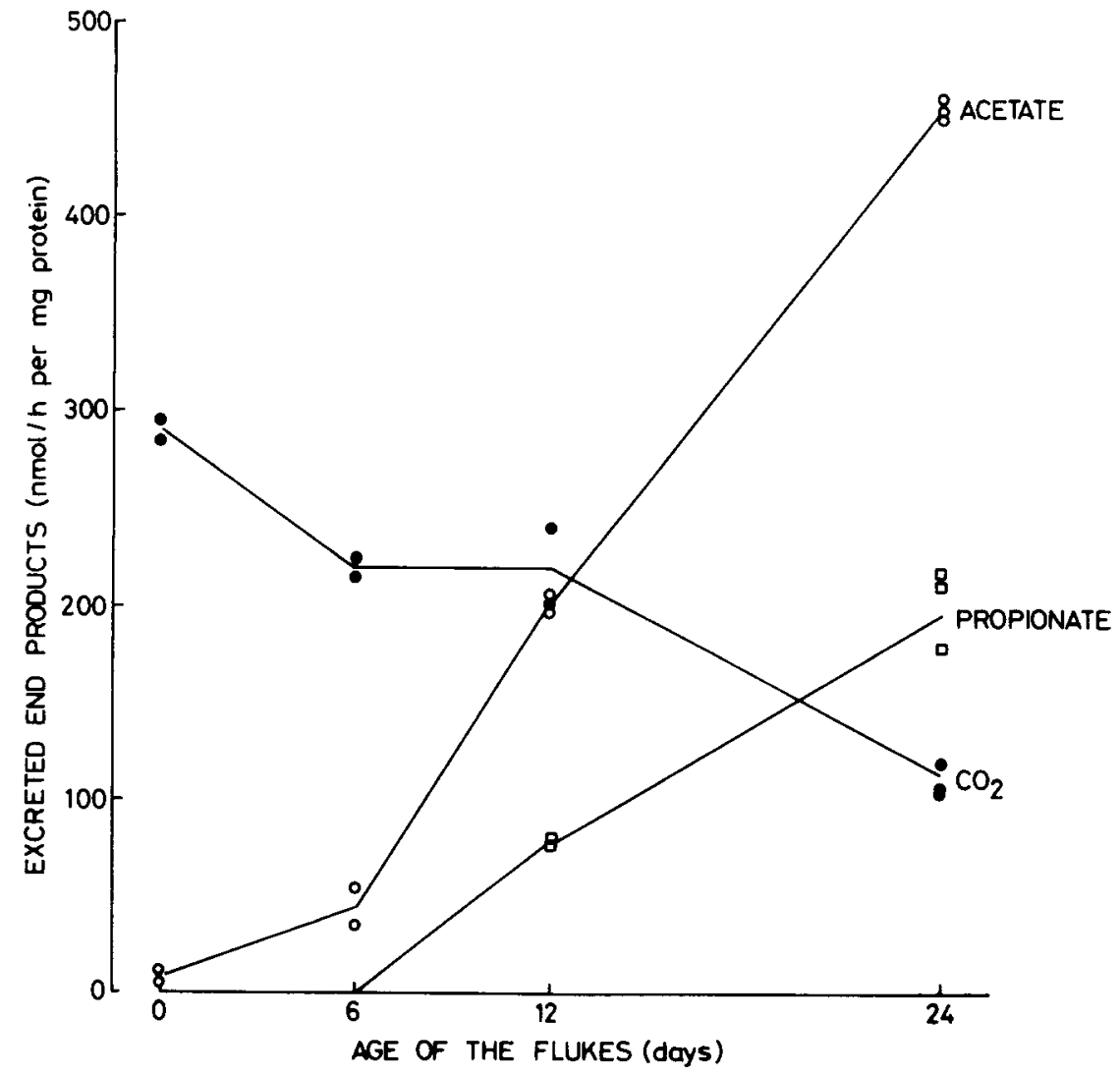

Fig. 1. Excreted end products in aerobic incubations of juvenile Fasciola hepatica of different ages. All values are corrected for blank incubations.

\section{TABLE IV}

The effect of fasting and refeeding on the glycogen content of juvenile $F$. hepatica

\begin{tabular}{lllll}
\hline Time & $\begin{array}{l}\text { Number of } \\
\text { flukes }\end{array}$ & $\begin{array}{l}\text { Glycogen } \\
\text { (ng/fluke) }\end{array}$ & $\begin{array}{l}\text { Protein } \\
\text { (ng/fluke) }\end{array}$ & $\begin{array}{l}\text { Glycogen } \\
\text { (ng/ng protein) }\end{array}$ \\
\hline Immediate & 1567 & 44.8 & 222 & 0.20 \\
& 1320 & 54.2 & 261 & 0.21 \\
After $5 \mathrm{~h}$ & 1730 & 29.8 & 224 & 0.13 \\
fasting & 2069 & 24.1 & 229 & 0.11 \\
After $5 \mathrm{~h}$ & & & & 0.28 \\
fasting +11 h refeeding & 1644 & 64.5 & 233 & 0.30 \\
\hline
\end{tabular}

Results of separate incubations are presented 
available. This is equivalent to the consumption of $105 \mathrm{nmol}$ of glucose per hour per $\mathrm{mg}$ protein, which is in reasonable agreement with the calculated rate of glucose consumption of $89 \pm 35 \mathrm{nmol} \cdot \mathrm{h}^{-1} \cdot \mathrm{mg}^{-1}$ in aerobic incubations of juveniles in the presence of $\mathrm{D}-\left[\mathrm{U}-{ }^{14} \mathrm{C}\right]$ glucose [2] and, though to a smaller degree, with the mean rate of $67 \mathrm{nmol}$ of glucose $\cdot \mathrm{h}^{-1} \cdot \mathrm{mg}^{-1}$ calculated from the aerobic incubations shown in Table II. Moreover, it seems unlikely that the juvenile liver fluke will degrade its internal glycogen stores in the presence of $11 \mathrm{mM}$ glucose since at this concentration of external glucose the fluke is able to replenish its glycogen stores (Table IV) and since this concentration is higher than the external glucose concentration in vivo (liver parenchyma). Therefore, the observed formation of labelled end products in the presence of $11 \mathrm{mM}$ glucose not only qualitatively reflects the pattern of glucose degradation but its rate is also a reliable indication of the true rate of end product formation in vitro.

Under aerobic conditions the in vitro excysted juveniles produced mainly carbon dioxide, whereas in the absence of oxygen they produced mainly propionate and acetate in a ratio of $2: 1$ (Table III). This result is in agreement with the results of incubations with $\mathrm{D}-\left[\mathrm{U}-{ }^{14} \mathrm{C}\right] \mathrm{glucose}$ which we published earlier [2] and it confirms that the in vitro excysted juvenile $F$. hepatica has an aerobic energy metabolism but is already fully equipped for anaerobic functioning.

The in vitro excysted juveniles as well as the in vivo developed flukes exhibited Krebs cycle activity, indicating that a functioning Krebs cycle is a true characteristic of all parenchymal stages of $F$. hepatica. During the parenchymal development of the liver fluke the Krebs cycle capacity slowly diminished. The production of labelled carbon dioxide decreased and acetate and propionate became the major end products (Fig. 1). The amounts of excreted acetate were much higher than half the amounts of excreted propionate and, therefore, the production of these end products was not in redox balance. In the production of acetate more NADH was reduced than was oxidized in the formation of propionate. The excess NADH had to be oxidized, for example via the respiratory chain. For this massive production of acetate, oxygen was indeed required, as is clear from the data in Table II: in the absence of oxygen a normal, adult-type, dismutation occurred.

In the adult the formation of $1 \mathrm{~mol}$ of acetate from acetyl-CoA is coupled with the synthesis of $1 \mathrm{~mol}$ of ATP [10]. If this is also the case in the developing juvenile, the net reaction of the production of acetate will be:

glucose $+4 \mathrm{ADP}+4 \mathrm{P}_{\mathrm{i}}+4 \mathrm{NAD}^{+} \rightarrow 2$ acetate $+2 \mathrm{CO}_{2}+4 \mathrm{ATP}+4 \mathrm{NADH}+4 \mathrm{H}^{+}$

$2 \mathrm{~mol}$ of $\mathrm{NADH}$ will be formed in the cytosol and, if the reduced equivalents are transported into the mitochondria via the glycerol 3-phosphate shuttle, they will give rise to the formation of $4 \mathrm{~mol}$ of ATP. The other $2 \mathrm{~mol}$ of NADH are formed inside the mitochondria and will yield another $6 \mathrm{~mol}$ of ATP when oxidized via the respiratory chain; this adds up to a total of $14 \mathrm{~mol}$ of ATP when $1 \mathrm{~mol}$ of glucose is degraded to $2 \mathrm{~mol}$ of acetate and $2 \mathrm{~mol}$ of carbon dioxide. The anaerobic dismutation leading to the pro- 
duction of propionate and acetate $(2: 1)$ will yield about 5 mol of ATP per degraded mol of glucose if it is assumed that the production of 1 mol of propionate from succinate is accompanied by the production of $1 \mathrm{~mol}$ of ATP [11-13]. The complete oxidation of glucose to carbon dioxide via the Krebs cycle will result in the formation of $36 \mathrm{~mol}$ of ATP per mol of glucose. These theoretical yields of ATP of the three pathways of glucose breakdown were used to calculate the contribution of each pathway to ATP synthesis in aerobic incubations of juvenile liver flukes at various stages of their development (Table V). These calculations were based on the assumptions that all the excreted propionate was formed by the anaerobic dismutation pathway and that this pathway produced propionate and acetate in a molar ratio of $2: 1$. Table $\mathrm{V}$ shows that in aerobic incubations of juveniles the contribution of the Krebs cycle to ATP synthesis slowly diminished during the whole development. It is also clear that the anaerobic dismutation started to contribute only at the later stages, but even after 24 days its contribution to ATP synthesis was not larger than that of the Krebs cycle. The most remarkable observation is that after 2 weeks of development the up to now unknown process of aerobic acetate formation became the most important energy source of the parenchymal liver fluke in vitro.

The contribution of the Krebs cycle to the total energy yield decreased, resulting in a decreased demand for oxygen by the Krebs cycle. On the other hand, the aerobic acetate formation also resulted in NADH oxidation via the respiratory chain. The amount of oxygen that had to be reduced by the respiratory chain at the various stages of the developing $F$. hepatica was calculated. Fig. 2 shows that the rate of oxygen reduction by the respiratory chain did not decrease during development. This demonstrates that the decreased Krebs cycle activity was not caused by a lack of oxygen, due to the growth of the fluke, nor by a decreased capacity of the respiratory chain. The transition from Krebs cycle activity to aerobic acetate formation may be caused either by a decreased Krebs cycle capacity or by an increased activity of the enzymes catalysing the conversion of acetyl-CoA into acetate; this problem is presently under investigation.

\section{TABLE V}

Contribution of the different pathways of glucose breakdown to ATP synthesis in juvenile $F$. hepatica under aerobic conditions

\begin{tabular}{lccc}
\hline \multirow{2}{*}{\begin{tabular}{l} 
Flukes \\
\cline { 2 - 3 }
\end{tabular}} & \multicolumn{2}{c}{ Calculated ATP synthesis (nmol/h per mg protein) } \\
\cline { 2 - 4 } & Krebs cycle & Aerobic acetate & Anacrobic dismutation \\
\hline In vitro excysted & $1740(97 \%)$ & $56(3 \%)$ & - \\
6 days old & $1317(81 \%)$ & $308(19 \%)$ & - \\
12 days old & $1323(48 \%)$ & $1138(41 \%)$ & $292(11 \%)$ \\
24 days old & $658(17 \%)$ & $2506(64 \%)$ & $740(19 \%)$ \\
\hline
\end{tabular}

These figures are the mean of those calculated from the results of the aerobic incubations shown in Fig. 1 . 


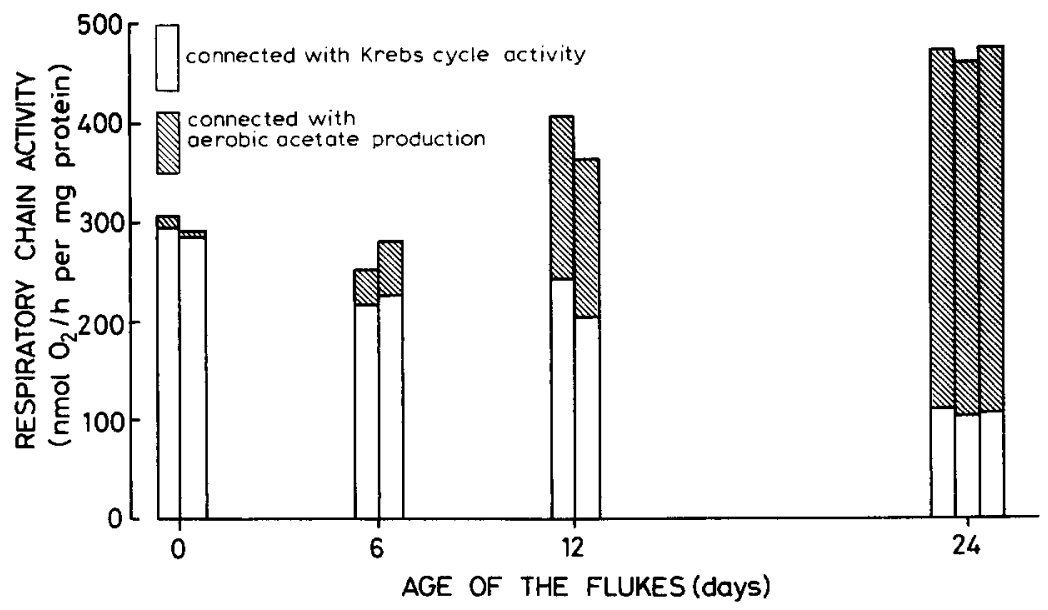

Fig. 2. The rate of oxygen reduction by the respiratory chain during development of the liver fluke, Fasciola hepatica. The values were calculated from the results of the aerobic incubations shown in Fig. 1.

The observed aerobic acetate formation may be a new target for anthelmintics, since it is a pathway not existing in the host whereas it is the most important energy source of the vulnerable, developing juvenile $F$. hepatica.

It should be noted that all data of aerobic in vitro incubations need not necessarily represent the in vivo situation, but reflect capacities which need not be utilized in situ. In our opinion, however, these data are indeed characteristic for the in vivo situation because oxygen availability in the liver parenchyma will not be rate-limiting. Our hypothesis, therefore, is that the liver fluke in vivo switches from Krebs cycle activity to the aerobic production of acetate since the activity of one or more of the Krebs cycle enzymes decreases. As soon as oxygen availability becomes the limiting factor, the parasite switches to the less economic anaerobic dismutation pathway of which the capacity is already present since its excystation out of the metacercarial cyst.

\section{ACKNOWLEDGEMENTS}

The authors thank Dr. H.J. Over and the Parasitology Department of the Central Veterinary Institute (Lelystad) for the generous supply of metacercariae and Mr. C.P.H. Gaasenbeek for expert technical assistance with the infection of rats and isolation of parenchymal liver flukes. This work was supported by the Netherlands Foundation for Chemical Research (S.O.N.) with financial aid from the Netherlands Organization for the Advancement of Pure Research (Z.W.O.).

\section{REFERENCES}

1 Lahoud, H., Prichard, R.K., McManus, W.R. and Schofield, P.J. (1971) Volatile fatty acid production by the adult liver fluke Fasciola hepatica. Comp. Biochem. Physiol. 38B, 379-391. 
2 Tielens, A.G.M., van der Meer, P. and van den Bergh, S.G. (1981) The aerobic energy metabolism of the juvenile Fasciola hepatica. Mol. Biochem. Parasitol. 3, 205-214.

3 Tielens, A.G.M., van der Meer, P. and van den Bergh, S.G. (1981) Fasciola hepatica: Simple, large-scale, in vitro excystment of metacercariae and the subsequent isolation of juvenile liver flukes. Exp. Parasitol. 51, 8-12.

4 Coles, G.C. (1972) Carbohydrate metabolism of larval Schistosoma mansoni. Int. J. Parasitol. 2, 341-352.

5 Susuki, S. (1931) Researches into the life history of Fasciola hepatica and its distribution in Formosa. J. Med. Assoc. Formosa, 30, $97-102$ (quoted by Dixon, K.E. (1966) Parasitology, 56, $431-456)$.

6 Boray, J.C. (1969) Experimental Fascioliasis in Australia. Adv. Parasitol. 7, 96-210.

7 Van den Bergh, S.G., van Vugt, F. and Tielens, A.G.M. (1980) Anaerobic and aerobic energy metabolism of the common liver fluke, Fasciola hepatica. In: Industrial and Clinical Enzymology, (Vitale, L. and Simeon, V. eds.), FEBS Symposia, Vol. 61, pp. 231 -242, Pergamon Press, Oxford.

8 Lowry, O.H., Rosebrough, N.J., Farr, A.L. and Randall, R.J. (1951) Protein measurement with the Folin reagent. J. Biol. Chem. 193, 265-275.

9 Hassid, W.Z. and Abraham, S. (1957) In: Methods in Enzymology (Colowick, S.P. and Kaplan, N.O., eds.), Vol. 3, p. 37, Academic Press, New York.

10 Van Vugt, F., van der Meer, P. and van den Bergh, S.G. (1979) The formation of propionate and acetate as terminal processes in the energy metabolism of the adult liver fluke Fasciola hepatica. Int. J. Biochem. 10, 11-18.

11 Köhler, P., Bryant, C. and Behm, C.A. (1978) ATP synthesis in a succinate decarboxylase system from Fasciola hepatica mitochondria. Int. J. Parasitol. 8, 399-404.

12 Van Vugt, F. (1980) The energy metabolism of the adult common liver fluke, Fasciola hepatica. Vet. Science Commun. 3, 299-316.

13 Pietrzak, S.M. and Saz, H.J. (1981) Succinate decarboxylation to propionate and the associated phosphorylation in Fasciola hepatica and Spirometra mansonoides. Mol. Biochem. Parasitol. 3, $61-70$. 